\section{Respuestas metabólicas inducidas por ejercicio físico de alta intensidad en mujeres sedentarias con glicemia basal alterada e hipercolesterolemia}

\author{
CRISTIAN ÁLVAREZ L. ${ }^{1, \mathrm{a}}$, RODRIGO RAMÍREZ-CAMPILLO ${ }^{2, \mathrm{~b}}$, \\ MARCELO FLORES O.,3,c, CARLOS HENRÍQUEZ-OLGUÍN ${ }^{5, \mathrm{~d}}$, \\ CHRISTIAN CAMPOS J.6,e, VANESA CARRASCO ${ }^{7, \mathrm{f}}$, \\ CRISTIAN MARTÍNEZ S. ${ }^{7, g}$, CARLOS CELIS-MORALES ${ }^{8, \mathrm{~h}}$
}

\section{Metabolic response to high intensity exercise training in sedentary hyper- glycemic and hypercholesterolemic women}

Background: High intensity training could be an effective way of improving health on individuals at high metabolic risk. Aim: To investigate the effects of a high intensity training intervention on metabolic-related markers in sedentary women at high metabolic risk. Material and Methods: Forty six sedentary women with a body mass index (BMI) over $25 \mathrm{~kg} / \mathrm{m}^{2}$ were assigned to four groups, according to their metabolic profile; hyperglycemia $(H, n=12)$, hyperglycemial hypercholesterolemia ( $H H, n=13)$, normoglycemia $(N, n=10)$ and normoglycemia/hypercholesterolemia ( $\mathrm{NH}, n=11)$. For 12 weeks and five days per week, subjects performed seven intervals of high intensity training (20 to 30 seconds) during a training session of 20 minutes. Anthropometric (body weight, body mass index (BMI), waist circumference) and metabolic variables (glucose, total cholesterol, $L D L, H D L$ and $T G$ ) were measured at baseline, at 6 and 12 weeks of intervention. Results: BMI and waist circumference decreased significantly after 12 weeks of intervention. Similarly, glucose decreased significantly after 12 weeks of intervention in all groups. The reduction was of higher magnitude in those groups with hyperglycemia $(H=-16 \%, H H=-22 \%$, $N=-7,5 \%, N H=-9,6 \%)$. However, lipid profile ( $T G$, total cholesterol, $L D L$ and $H D L)$ improved significantly only in the hypercholesterolemic groups. Conclusions: Physical activity programs incorporating high intensity training can improve glucose and lipid profile in women with metabolic disorders. Moreover, this benefit is greatest in those individuals with highest metabolic burden.

(Rev Med Chile 2013; 141: 1293-1299).

Key words: Exercise; Diabetes mellitus; Hypercholesterolemia.
'Centro de Salud Familiar de Los Lagos, Región de Los Ríos, Chile.

2Departamento Ciencias de la Actividad Física, Universidad de Los Lagos, Osorno, Chile.

${ }^{3}$ Servicio de Kinesiología, Hospital de Carabineros de Chile

${ }^{4}$ Facultad de Medicina, Departamento de Fisiología del Ejercicio, Universidad de Melbourne, Melbourne, Australia.

5Unidad de Fisiología del Ejercicio, Laboratorio de Ciencias del Ejercicio, Clínica MEDS

Santiago, Chile.

${ }^{6}$ Carrera de Kinesiología. Unidad Docente Asociada Ciencias de la Salud. Escuela de Medicina. Pontificia Universidad Católica de Chile.

${ }^{7}$ Facultad de Educación, Ciencias Sociales y Humanidades, Departamento de Educación Física, Universidad de la Frontera, Temuco, Chile.

${ }^{8}$ Centro de Investigación en Nutrición Humana, Instituto de Investigación en Envejecimiento y Salud, Universidad de Newcastle, Inglaterra.

aProfesor de Educación Física, Lic., MSc. Entrenamiento Deportivo.

bProfesor de Educación Física, Lic., MSc. Fisiología del Ejercicio.

'Kinesiólogo, Lic., MSc Fisiología del Ejercicio dProfesor de Educación Física, Lic., MSc.

Ciencias Biológicas.

eKinesiólogo, Lic., MSc., PhD en Actividad Física y Salud.

fProfesora de Educación Física, MSc. Educación Física.

9Profesor de Educación Física, MSc Motricidad Humana.

${ }^{\text {h}}$ Doctor en Ciencias Cardiovasculares y Biomédicas (PhD).

\section{Financiamiento: Este proyecto de} investigación fue financiado por el concurso de Fondos del Departamento de Salud Pública Municipal de la llustre Municipalidad de Los Lagos y el Departamento de Ciencias de la Actividad Física de la Universidad de Los Lagos. No se declaran conflictos de intereses entre las fuentes de financiamiento en el diseño del presente estudio.

Recibido el 28 de marzo de 2013, aceptado el 23 de julio de 2013

Correspondencia a:

Dr. Carlos Celis-Morales

Biomedical Research Building

Campus for Ageing and Vitality

Newcastle Upon Tyne,

NE4 5PL. UK.

Teléfono: 44-01912481141

Email:carlos.celis@ncl.ac.uk 
L a Encuesta Nacional de Salud (ENS, 2010), reportó que $10 \%$ de las mujeres chilenas son diabéticas y $38 \%$ sufren de hipercolesterolemia $^{1}$, ambas asociadas al desarrollo de enfermedad cardiovascular $(\mathrm{ECV})^{2}$. Adicionalmente, altos niveles de obesidad (64\%) y sedentarismo (93\%) han sido identificados como algunos de los principales factores de riesgo de estas condiciones en población chilena ${ }^{1,3}$.

Considerando el presupuesto invertido en el tratamiento farmacológico de estas condiciones ${ }^{4}$, se hace relevante el investigar sobre los beneficios de tratamientos alternativos como el ejercicio físico (EF). A pesar de que recomendaciones de EF basadas en actividades aeróbicas de baja intensidad ${ }^{5} y$ sobrecarga ${ }^{6}$ han reportado importantes beneficios en el manejo de alteraciones metabólicas, una de las principales razones entregadas por la población para no cumplir con las recomendaciones de actividad física para la salud pública (150-300 min/ semana) es la "falta de tiempo"7. Poco se conoce respecto a los efectos de otros tipos de EF como el ejercicio intervalado de alta intensidad (HIT del inglés high intensity interval training), propuesto como una metodología tiempo-eficiente para el tratamiento de alteraciones metabólicas ${ }^{8,9}$.

El objetivo del presente estudio fue investigar los efectos de un programa guiado de EF de alta intensidad sobre variables antropométricas y metabólicas en mujeres sedentarias con glicemia basal alterada e hipercolesterolemia.

\section{Métodos}

En un estudio de diseño experimental, cincuenta y nueve mujeres, pre-menopáusicas con bajo nivel de actividad física (menos de $150 \mathrm{~min}$ de actividad física a la semana $)^{10}$, usuarias del centro de salud familiar de Los Lagos (CESFAM), Región de Los Ríos, Chile, fueron invitadas a participar en un programa guiado de EF.

Los criterios de inclusión fueron: mujeres sedentarias, pre-menopáusicas, índice de masa corporal (IMC $>25$ a $35 \mathrm{~kg} / \mathrm{m}^{2}$ ), glicemia $<100$ $\mathrm{mg} / \mathrm{dl}$ (normo-glicémicos) y 100 a $126 \mathrm{mg} / \mathrm{dl}$ (glicemia basal alterada $(\mathrm{GBA}))^{11}$, colesterol total (CT) $<200 \mathrm{mg} / \mathrm{dl}$ (normo-colesterolémicos) y > 200 $\mathrm{mg} / \mathrm{dl}$ (hiper-colesterolémicos) ${ }^{2}$, con control médico y ECG actualizado. Se excluyeron sujetos con problemas ósteo-articulares, isquemia, arritmia, taquicardia o EPOC, con tratamiento farmacológico que afectara el peso corporal o control metabólico de la glucosa y lípidos (sujetos excluidos $n=13$ ). Basado en estudios previos ${ }^{12}$, el tamaño de la muestra se estimó con cambios observados en glicemia post intervención de $6,1 \mathrm{mg} / \mathrm{dl}$ y una desviación estándar de 5,6. Una muestra de 9 participantes por grupo (total $\mathrm{n}=36$ ) nos otorga un $\alpha=0,05$ y $\beta=0,80$. En este estudio cuarenta y seis participantes cumplieron con los criterios de inclusión y fueron asignados a cuatro grupos: GBA $(\mathrm{H}, \mathrm{n}=12), \mathrm{GBA} /$ hiper-colesterolémicas $(\mathrm{HH}, \mathrm{n}=13)$, normo-glicémicas $(\mathrm{N}, \mathrm{n}=10) \mathrm{y}$ normo-glicémicas/hiper-colesterolémicas $(\mathrm{NH}, \mathrm{n}$ =11). Todos los sujetos dieron su consentimiento informado por escrito. El estudio fue desarrollado de acuerdo a la Declaración de Helsinki y aprobado por el comité de ética del CESFAM Los Lagos, Chile.

Las mediciones de peso corporal, talla, IMC, perímetro de cintura (PC), se realizaron preintervención, al finalizar seis y doce semanas de intervención. Paralelamente, se midieron en ayuna las variables plasmáticas de glicemia, colesterol total (CT), colesterol HDL, y triglicéridos (TG). Se instruyó a todos los sujetos no modificar sus hábitos de alimentación y/o actividad física durante la intervención.

\section{Evaluación antropométrica}

La talla se midió con un estadiómetro profesional (Health o Meter ${ }^{\varpi}$, USA), con precisión de $0,1 \mathrm{~cm}$. El peso corporal se midió con una balanza digital con precisión de $0,01 \mathrm{~kg}\left(\mathrm{OMRON}^{\varpi}\right.$, Model HBF-INT, Alemania). El IMC se calculó en base al peso corporal dividido por la talla al cuadrado $(\mathrm{kg} /$ $\mathrm{m}^{2}$ ). El PC se midió con una cinta métrica con una precisión de 0,1 cm (Hoechstmass ${ }^{\circledR}$, Alemania). Las evaluaciones se realizaron utilizando el protocolo estandarizado de la Sociedad Internacional para el Avance de la Cineantropometría (ISAK) ${ }^{13}$.

\section{Evaluación de glicemia y lípidos plasmáticos}

Posterior a $12 \mathrm{~h}$ de ayuno, se obtuvieron muestras $(4 \mathrm{ml})$ de sangre entre las 08:00 am y 10:00 am. Las muestras fueron inmediatamente colocadas en hielo y centrifugadas a $3.000 \mathrm{rpm}$. durante 15 min a $4^{\circ} \mathrm{C}$. Posteriormente, el plasma se almacenó a- $20^{\circ} \mathrm{C}$ hasta su análisis. Glicemia, TG, $\mathrm{CT}$, colesterol HDL (C-HDL) fueron analizados por métodos estándar, similar a otros estudios ${ }^{14}$. Se 
analizó la glicemia a través del método enzimático con la técnica glucosa-oxidasa (Trinder, Genzyme Diagnostics, Canada). CT, TGy C-HDL se analizaron mediante el método enzimático calorimétrico (Diagnostica mbh, Alemania). El colesterol LDL (C-LDL) se calculó con la fórmula de Friedewald ${ }^{15}$.

\section{Programa de EF de alta intensidad (HIT)}

Todos los grupos completaron 12 semanas de $\mathrm{EF}$, con frecuencia de 5 sesiones/semana, $20 \mathrm{~min} /$ sesión y supervisados por un educador físico. En cada sesión, se realizaron 2 min de calistenia, y 7 intervalos de carrera $\geq 90 \%$ de la frecuencia cardiaca de reserva (FCR) determinada mediante la ecuación de Karvonen ${ }^{16}$ y controlada mediante un cardiómetro (Polar ${ }^{\circledR}$ modelo RS400, Finlandia), que cada sujeto usó para controlar su intensidad de ejercicio. Cada intervalo de alta intensidad fue alternado con 2 min de pausa activa (caminata). Fueron excluidos todos los participantes $(n=4)$ con una asistencia menor a $70 \%$ de las sesiones. Las características del programa se presentan en la (Tabla 1).

El volumen, intensidad y densidad del programa de EF consideró intervalos de alta intensidad con duración de 20s, con pausa de recuperación activa de 120s. Posteriormente, cada 2 semanas, la duración de cada intervalo se incrementó en $10 \%$ y el descanso activo se redujo en 2 s respecto al tiempo inicial, a modo de mantener la carga de EF declarada.

\section{Análisis estadístico}

Se utilizó media \pm error estándar de la media para describir las variables cuantitativas. Se uti- lizó el test de Shapiro-Wilk para determinar la normalidad de los datos. Los efectos de la intervención se analizaron con un análisis de varianza usando la técnica General Linear Model (GLM). Bonferroni post hoc fue aplicado para localizar las diferencias entre los tiempo de medición (pre, 6 semanas y 12 semanas $24 \mathrm{~h}$ y $48 \mathrm{~h}$ ) y grupos de intervención $(\mathrm{H}, \mathrm{HH}, \mathrm{NH}, \mathrm{N})$. El nivel para significancia estadística se estableció en $p \leq 0,05$. Todos los análisis estadísticos se realizaron utilizando el software STATISTICA (Versión 8.0, StatSoft, Inc, Oklahoma, USA).

\section{Resultados}

La adherencia al programa de EF por cada grupo de estudio fue: $\mathrm{H}(83 \%), \mathrm{HH}(77 \%), \mathrm{N}$ (100\%), y NH (91\%). Las características de los grupos de investigación se presentan en la Tabla 2. La edad promedio de los participantes presentó diferencias significativas entre grupos. No obstante, no se encontraron diferencias en peso, IMC, PC, C-HDL, C-LDL y TG entre los grupos pre-intervención. Sólo CT y glicemia presentan diferencias pre-intervención. No obstante, estas diferencias están en concordancia con el diseño del estudio, ya que mujeres con niveles alterados de glicemia y colesterol fueron reclutadas en los grupos $\mathrm{H}, \mathrm{HH}$ y $\mathrm{NH}$ (Tabla 2).

A las 6 y 12 semanas de intervención todos los grupos presentaron reducciones significativas en peso corporal, IMC y PC. Estas diferencias se mantuvieron al ajustar los análisis por edad (Tabla 2). La glicemia disminuyó significativamente a las 6, y

Tabla 1. Descripción del programa de entrenamiento de alta intensidad

\begin{tabular}{|lcccccc|}
\hline Variable & $\begin{array}{c}\text { Semana } \\
\mathbf{1 - 2}\end{array}$ & $\begin{array}{c}\text { Semana } \\
\mathbf{3 - 4}\end{array}$ & $\begin{array}{c}\text { Semana } \\
\mathbf{5 - 6}\end{array}$ & $\begin{array}{c}\text { Semana } \\
\mathbf{7 - 8}\end{array}$ & $\begin{array}{c}\text { Semana } \\
\mathbf{9 - 1 0}\end{array}$ & $\begin{array}{c}\text { Semana } \\
\mathbf{1 1 - 1 2}\end{array}$ \\
\hline Intensidad c/intervalo & $100 \% \mathrm{FCR}$ & $100 \% \mathrm{FCR}$ & $100 \% \mathrm{FCR}$ & $100 \% \mathrm{FCR}$ & $100 \% \mathrm{FCR}$ & $100 \% \mathrm{FCR}$ \\
\hline Duración intervalo de alta intensidad (s) & 20 & 22 & 24 & 26 & 28 & 30 \\
\hline Recuperación (s) & 120 & 118 & 116 & 114 & 112 & 110 \\
Método de recuperación & Caminata & Caminata & Caminata & Caminata & Caminata & Caminata \\
\hline No total intervalos & 7 & 7 & 7 & 7 & 7 & 7 \\
Volumen/día (min) & 20 & 20 & 20 & 20 & 20 & 20 \\
\hline Volumen/sem (min) & 100 & 100 & 100 & 100 & 100 & 100 \\
\hline
\end{tabular}

FCR: Frecuencia cardiaca de reserva. 
Tabla 2. Efectos del programa de ejercicio físico de alta intensidad sobre indicadores antropométricos

\begin{tabular}{|c|c|c|c|c|c|c|}
\hline Variable & Test & $\begin{array}{c}H \\
(n=12)\end{array}$ & $\begin{array}{c}\text { HH } \\
(n=13)\end{array}$ & $\begin{array}{c}\text { NH } \\
(n=11)\end{array}$ & $\begin{array}{c}N \\
(n=10)\end{array}$ & P-interacción \#\# \\
\hline Edad & Pre $_{0}$ & $40,0 \pm 7,0^{\mathrm{NH}, \mathrm{N}}$ & $40,1 \pm 6,0^{\mathrm{NH}, \mathrm{N}}$ & $35,3 \pm 10,0^{\mathrm{H}, \mathrm{HH}}$ & $34,6 \pm 7,5^{\mathrm{H}, \mathrm{HH}}$ & 0,012 \\
\hline Talla (m) & Pre $_{0}$ & $1,55 \pm 0,1$ & $1,56 \pm 0,1$ & $1,59 \cdot 0,1$ & $1,58 \pm 0,2$ & 0,199 \\
\hline \multirow[t]{3}{*}{ Peso (kg) } & Pre $_{0}$ & $69,9 \pm 2,7$ & $72,8 \pm 2,7$ & $67,7 \pm 2,4$ & $72,1 \pm 2,7$ & 0,510 \\
\hline & Post 6s & $67,9 \pm 2,1^{*}$ & $71,7 \pm 2,5$ & $66,4 \pm 2,3$ & $70,6 \pm 2,3$ & \\
\hline & Post $12 \mathrm{~s}$ & $66,3 \pm 2,8^{*}$ & $70,5 \pm 2,0^{¥}$ & $64,6 \pm 2,0^{¥}$ & $69,6 \pm 2,5^{¥}$ & \\
\hline $\begin{array}{l}\text { IMC } \\
\left(\mathrm{kg} / \mathrm{m}^{2}\right)\end{array}$ & $\begin{array}{l}\text { Pre }_{0} \\
\text { Post } 6 \mathrm{~s} \\
\text { Post 12s }\end{array}$ & $\begin{array}{l}29,2 \pm 1,0 \\
28,3 \pm 1,2^{*} \\
27,9 \pm 1,3^{*}\end{array}$ & $\begin{array}{l}30,1 \pm 1,1 \\
29,7 \pm 1,3 \\
29,1 \pm 0,9^{*}\end{array}$ & $\begin{array}{l}28,2 \pm 1,4 \\
27,1 \pm 0,8^{*} \\
27,0 \pm 0,9^{*}\end{array}$ & $\begin{array}{l}27,0 \pm 1,1 \\
26,4 \pm 1,0 \\
26,1 \pm 1,2^{*}\end{array}$ & 0,244 \\
\hline $\begin{array}{l}\text { Perímetro } \\
\text { cintura } \\
(\mathrm{cm})\end{array}$ & $\begin{array}{l}\text { Pre }_{0} \\
\text { Post 6s } \\
\text { Post 12s }\end{array}$ & $\begin{array}{l}97,5 \pm 2,3 \\
94,0 \pm 2,1^{*} \\
91,8 \pm 2,3^{*}\end{array}$ & $\begin{array}{r}100,5 \pm 2,3 \\
97,8 \pm 2,2^{*} \\
95,9 \pm 2,4^{*}\end{array}$ & $\begin{aligned} 101,6 & \pm 2,3 \\
100,1 & \pm 2,0 \\
97,5 & \pm 2,3^{*}\end{aligned}$ & $\begin{array}{l}97,2 \pm 2,4 \\
95,1 \pm 2,0^{*} \\
93,6 \pm 2,1^{*}\end{array}$ & 0,507 \\
\hline
\end{tabular}

Los datos son presentados como media \pm error estándar de la media. Las diferencias entre los tiempos de evaluación fueron estimadas mediante General Linear Model y ajustadas por edad. Diferencias significativas $(p>0,05)$ entre tiempos de evaluación se señalaron con los siguientes símbolos ( ${ }^{*} \operatorname{Pre}_{0}$ vs Post 6 semanas; ${ }^{*}$ Pre $_{0}$ vs Post 12 semanas. \#\#Diferencias significativas entre grupos Pre intervencion fueron señalados con los codigos de los grupos intervenidos (HH: Glicemia Basal Alterada/Hipercolesterolémicos; H: Glicemia Basal Alterada; NH: Normo-glicémicos/Hiper-colesterolémicos; N: Normo-glicémicos).

Tabla 3. Efectos del programa de ejercicio físico de alta intensidad sobre marcadores metabólicos de glicemia y perfil lipídico

\begin{tabular}{|c|c|c|c|c|c|c|}
\hline & Test & $H(n=12)$ & $H H(n=13)$ & NH $(n=11)$ & $N(n=10)$ & $\begin{array}{l}\text { P-inter- } \\
\text { acción\#\# }\end{array}$ \\
\hline $\begin{array}{l}\text { Glicemia } \\
\text { (mg/dl) }\end{array}$ & $\begin{array}{l}\text { Pre }_{0} \\
\text { Post 6s } \\
\text { Post 12s }(24 \mathrm{~h}) \\
\text { Post 12s }(48 \mathrm{~h})\end{array}$ & $\begin{array}{c}112,1 \pm 2,0^{\mathrm{NH}, \mathrm{N}} \\
103,0 \pm 1,2 \\
94,1 \pm 1,1^{*} \\
96,5 \pm 2,6^{\#}\end{array}$ & $\begin{array}{c}114,9 \pm 1,8^{\mathrm{NH}, \mathrm{N}} \\
104,2 \pm 2,4 \\
92,5 \pm 1,8^{¥} \\
94,0 \pm 2,1^{\#}\end{array}$ & $\begin{array}{c}94,2 \pm 1,1^{\mathrm{H}, \mathrm{HH}} \\
90,1 \pm 1,5 \\
85,1 \pm 2,1^{¥} \\
86,9 \pm 2,5^{\#}\end{array}$ & $\begin{array}{c}92,3 \pm 1,6^{\mathrm{H}, \mathrm{HH}} \\
89,1 \pm 1,7 \\
85,3 \pm 1,5^{¥} \\
87,0 \pm 2,1\end{array}$ & 0,002 \\
\hline $\begin{array}{l}\text { Colesterol } \\
\text { total } \\
(\mathrm{mg} / \mathrm{dl})\end{array}$ & $\begin{array}{l}\text { Pre }_{0} \\
\text { Post } 6 s \\
\text { Post 12s }(24 \mathrm{~h}) \\
\text { Post 12s }(48 \mathrm{~h})\end{array}$ & $\begin{array}{c}167,9 \pm 6,0^{\mathrm{HH}, \mathrm{NH}} \\
164,1 \pm 6,1 \\
159,3 \pm 6,4 \\
162,9 \pm 6,1\end{array}$ & $\begin{array}{c}222,9 \pm 6,1^{\mathrm{H}, \mathrm{N}} \\
212,2 \pm 6,0 \\
194,1 \pm 6,2^{7} \\
196,2 \pm 6,3^{\#}\end{array}$ & $\begin{array}{c}220,7 \pm 5,8^{H, N} \\
206,6 \pm 4,3 \\
193,8 \pm 3,5^{¥} \\
195,1 \pm 4,1^{\#}\end{array}$ & $\begin{array}{c}173,2 \pm 7,6 \mathrm{HH,NH} \\
170,5 \pm 8,2 \\
168,1 \pm 8,1 \\
170,6 \pm 6,8\end{array}$ & 0,001 \\
\hline $\begin{array}{l}\text { LDL } \\
(\mathrm{mg} / \mathrm{dl})\end{array}$ & $\begin{array}{l}\text { Pre }_{0} \\
\text { Post 6s } \\
\text { Post 12s } \\
\left.\text { Post 12s } s_{(48)} \mathrm{h}\right)\end{array}$ & $\begin{array}{c}102,6 \pm 7,2^{\mathrm{HH}, \mathrm{NH}, \mathrm{N}} \\
96,6 \pm 8,5 \\
92,0 \pm 8.9 \\
93,2 \pm 8.2\end{array}$ & $\begin{array}{c}138,1 \pm 8,1^{\mathrm{H}, \mathrm{N}} \\
128,2 \pm 7,7 \\
113,7 \pm 7,6^{¥} \\
112,2 \pm 8,2^{\#}\end{array}$ & $\begin{array}{c}136,4 \pm 6,8^{H, N} \\
131,1 \pm 6,5 \\
117,9 \pm 6,2^{7} \\
118,6 \pm 6,5^{\#}\end{array}$ & $\begin{array}{c}120,3 \pm 8,9 \mathrm{H}, \mathrm{HH}, \mathrm{NH} \\
117,0 \pm 7,7 \\
111,3 \pm 7,9 \\
113,1 \pm 8,1\end{array}$ & 0,004 \\
\hline $\begin{array}{l}\mathrm{HDL} \\
(\mathrm{mg} / \mathrm{dl})\end{array}$ & $\begin{array}{l}\text { Pre }_{0} \\
\text { Post 6s } \\
\text { Post 12s }{ }_{(24 \mathrm{~h})} \\
\text { Post 12s }{ }_{(48 \mathrm{~h})}\end{array}$ & $\begin{array}{c}58,7 \pm 4,0^{\mathrm{NH}, \mathrm{N}} \\
61,8 \pm 4,5 \\
65,2 \pm 4,7^{¥} \\
64,3 \pm 4,0^{\#}\end{array}$ & $\begin{array}{c}56,2 \pm 4,0^{\mathrm{NH}} \\
61,6 \pm 4,3 \\
69,8 \pm 4,4^{7} \\
67,2 \pm 4,7^{\#}\end{array}$ & $\begin{array}{c}46,4 \pm 3,7^{\mathrm{H}, \mathrm{HH}} \\
50,4 \pm 3,5 \\
56,7 \pm 3,4^{¥} \\
55,1 \pm 3,7^{\#}\end{array}$ & $\begin{array}{c}50,8 \pm 3,4^{H} \\
52,4 \pm 4,6 \\
57,1 \pm 4,7^{*} \\
56,8 \pm 3,6^{\#}\end{array}$ & 0,023 \\
\hline $\begin{array}{l}\text { Triglicéridos } \\
(\mathrm{mg} / \mathrm{dl})\end{array}$ & $\begin{array}{l}\text { Pre }_{0} \\
\text { Post 6s } \\
\text { Post 12s }(24 \mathrm{~h}) \\
\text { Post 12s }\end{array}$ & $\begin{array}{c}118,3 \pm 10,9 \mathrm{HH}, \mathrm{NH}, \mathrm{N} \\
111,2 \pm 11,1 \\
106,3 \pm 11,9 \\
107,8 \pm 14,2\end{array}$ & $\begin{array}{c}138,6 \pm 13,1^{H, N H} \\
130,2 \pm 12,8 \\
118,3 \pm 12,7^{*} \\
121,9 \pm 13,2^{\#}\end{array}$ & $\begin{array}{c}150,8 \pm 12,4^{\mathrm{H}, \mathrm{HH}, \mathrm{N}} \\
146,9 \pm 12,1 \\
132,7 \pm 12,6^{\sharp} \\
133,5 \pm 12,9^{\#}\end{array}$ & $\begin{array}{c}139,6 \pm 15,4^{\mathrm{H}, \mathrm{NH}} \\
135,8 \pm 15,2 \\
127,6 \pm 11,7 \\
129,1 \pm 14,2\end{array}$ & 0,012 \\
\hline
\end{tabular}

Los datos son presentados como media \pm error estándar de la media. Las diferencias entre los tiempos de evaluación fueron estimadas mediante General Linear Models y ajustados por edad, IMC y perímetro de cintura, Diferencias significativas $(p>0,05)$ entre tiempos de evaluación se señalaron con los siguientes símbolos ( ${ }^{*} \operatorname{Pre}_{0}$ vs Post 6 semanas; ${ }^{*}$ Pre $_{0}$ vs Post 12 semanas (24 h);\# Pre $_{0}$ vs Post 12 semanas (48 h). \#\#iferencias significativas entre grupos Pre intervencion fueron señalados con los codigos de los grupos intervenidos (HH: Glicemia Basal Alterada/Hiper-colesterolémicos; H: Glicemia Basal Alterada; NH: Normo-glicémicos/ Hiper-colesterolémicos; N: Normo-glicémicos). 
12 semanas de intervención (a 24 h y 48 h) en todos los grupos, excepto en el grupo normo-glicémico $(\mathrm{N})$, en el cual sólo se observa una disminución significativa en la semana 12 ( $24 \mathrm{~h}$ ) (Tabla 3). Los niveles de CT sólo disminuyeron significativamente en los grupos con hiper-colesterolemia ( $\mathrm{HH}$ y $\mathrm{NH}$ ) a las 12 semanas ( $24 \mathrm{~h}$ y $48 \mathrm{~h}$ ). Similarmente, se observó una disminución del C-LDL y TG en todos los grupos, pero esta sólo fue significativa en aquellos con hiper-colesterolemia ( $\mathrm{HH}$ y $\mathrm{NH})$.
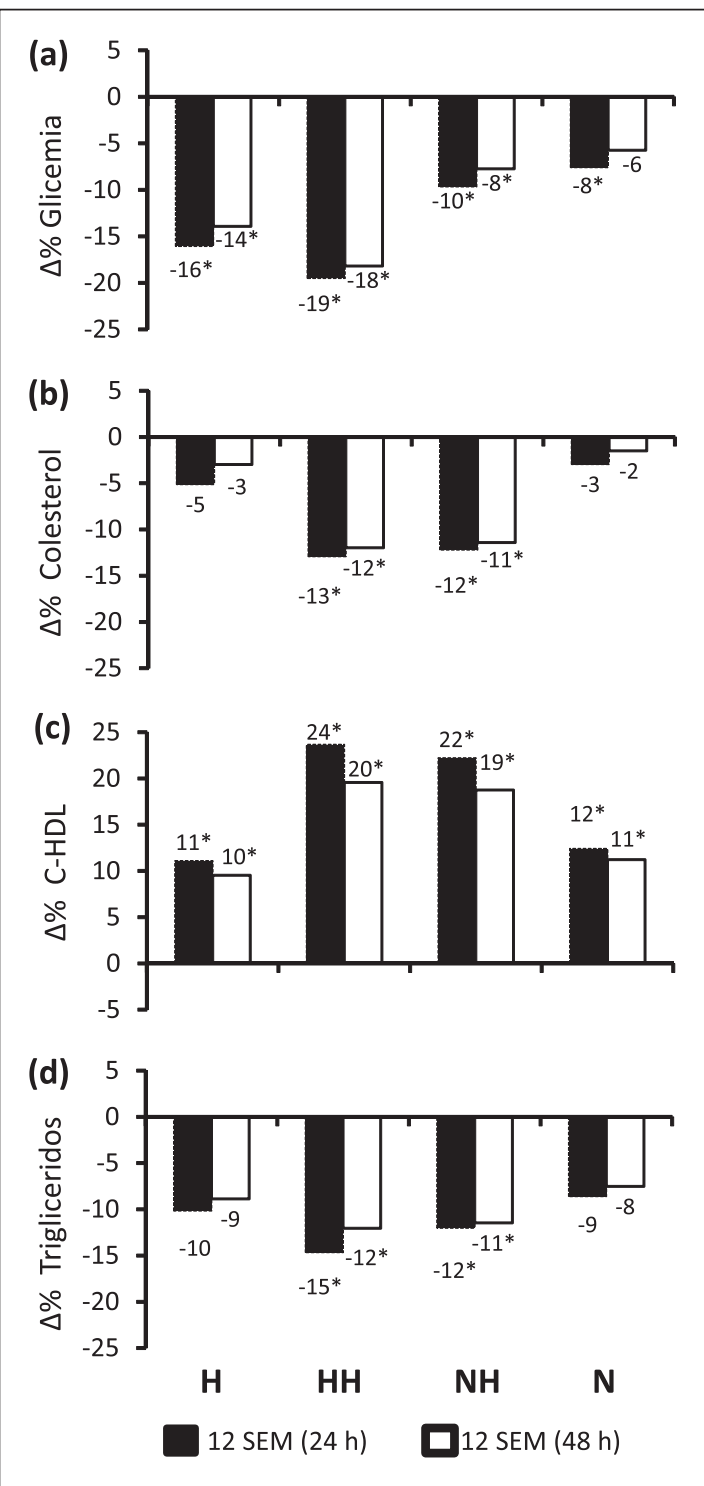

Figura 1. Modificación de la glicemia y perfil de lípidos posterior a 24 y $48 \mathrm{~h}$ de finalizada la intervención.
Mientras que el C-HDL aumentó significativamente en todos los grupos (Tabla 3 y Figura 1). Todos estos resultados en marcadores metabólicos fueron ajustados por edad, y cambios en IMC y PC durante la intervención (Tabla 3 y Figura 1).

\section{Discusión}

Los principales resultados de este estudio indican que 12 semanas de EF de alta intensidad y corta duración mejoran substancialmente los niveles de glicemia y C-HDL en mujeres con y sin riesgo metabólico. Adicionalmente, el programa otorga beneficios extras a aquellos grupos con perfil lipídico alterado, los cuales presentan una mejora significativa en CT, C-LDL y TG. Estos resultados sugieren que la implementación de EF de alta intensidad es efectiva para la reducción de factores de riesgo cardiovascular en mujeres chilenas.

En relación a las modificaciones producidas en peso corporal, IMC y PC post-intervención, se debe señalar que estas fluctuaron entre $-3 \%$ a $-5 \%$, $-3,3 \%$ a $-4,4 \%$ y $-3,7 \%$ a $-5,8 \%$, respectivamente. Estos resultados concuerdan con estudios previos donde una intervención con EF de alta intensidad redujo los niveles de IMC y PC en $-3,8 \%$ y $-4,9$, respectivamente ${ }^{17}$.

Interesantemente, después de 12 semanas de intervención las reducciones en los niveles de glicemia (a 24 h finalizada la intervención) variaron entre $-7,5 \%$ y $-22,4 \%$. Estas reducciones fueron de mayor impacto en aquellos grupos que presentaban alterados niveles de glicemia preintervención (H: $-16 \%, \mathrm{HH}:-22,4 \%, \mathrm{NH}:-9,6 \%$, $\mathrm{N}:-7,5 \%)$. A $48 \mathrm{~h}$ posterior al fin de la intervención, los niveles de glicemia aumentaron entre $1,4 \%$ y $2,5 \%$ en comparación a las mediaciones realizadas a $24 \mathrm{~h}$ post-intervención. Esto indica que las reducciones logradas por el programa de ejercicio físico son más bien de tipo crónico y no agudo. Esto concuerda con estudios anteriores realizados en mujeres pre diabéticas donde las mejoras en los niveles de glicemia se mantenían $72 \mathrm{~h}$ post intervención ${ }^{12}$. Adicionalmente, se puede señalar que las mejoras en los niveles de glicemia post-intervención podrían ser explicadas por estudios previos $^{18}$ que muestran los efectos del EF de alta intensidad sobre la activación de vías de señalización intracelularmediadas por la proteína 
kinasa activada por AMP (AMPK), enzima clave en el metabolismo de glucosa y lípidos, cuyo efecto mimetiza el efecto de la insulina induciendo la translocación de los transportadores de glucosa Glut-4 hacia la superficie celular, lo que finalmente aumenta la captación de glucosa desde la sangre hacia el músculo esquelético. Además, otros efectos biológicos se han reportado como respuestas a este tipo de entrenamiento, entre los que se destacan un aumento en la biogénesis mitocondrial y la capacidad oxidativa de las fibras musculares. Esto último se ha relacionado con la expresión del factor de transcripción PGC-1 $\alpha$ post intervención con EF de alta intensidad ${ }^{19}$.

En relación a los efectos de la intervención sobre el perfil lipídico, cabe destacar que todos los grupos presentaron mejoras en estas variables. No obstante los cambios sólo fueron significativos en aquellos grupos con hiper-colesterolemia. La magnitud de estos cambios fue de $-28 \%$ y $-26,9 \%$ en CT, $-24 \%$ y $-18 \%$ en C-LDL, $+13 \%$ y $+10 \%$ en CHDL, $-20 \%$ y $-18 \%$ en TG en los grupos $\mathrm{HH}$ y NH, respectivamente. Estos cambios son superiores a los reportados en estudios previos por Balducci y col., ${ }^{17}$ (-12\% glicemia, -11\% CT, $-20 \%$ C-LDL, $+6,5 \%$ C-HDL y -2,6\% TG). No obstante, esta diferencia en los cambios podría estar explicada por las características de los participantes, ya que la intervención de Balducci y col. ${ }^{17}$ fue aplicada en personas sin alteraciones metabólicas. Como ya se ha reportado anteriormente ${ }^{20,21}$ y como también lo demuestran nuestros resultados, los grupos con mejor respuesta al EF son aquellos que presentan una mayor alteración en sus parámetros metabólicos al inicio del programa de EF, sugiriendo que mientras menor es la condición física y mayor el riesgo metabólico, los beneficios alcanzados mediante el EF serán más significativos.

Aspectos que podrían ser una limitante dentro del estudio son los cambios producidos en peso corporal, IMC y PC post intervención, los cuales podrían explicar las mejoras en los parámetros metabólicos. No obstante, al correlacionar los cambios en peso corporal, IMC y PC con cambios en parámetros metabólicos, se observó una correlación significativa sólo con CT. Por ello, los análisis estadísticos para marcadores metabólicos fueron ajustados a diferencias en edad, cambios en IMC, peso corporal y PC. Otra limitante, podría relacionarse a cambios en los patrones de actividad física de los participantes. Si bien no se estimó consumo máximo de oxígeno, se instruyó a los participantes mantener sus estilos de vida. Debido a que los participantes reclutados eran sedentarios, sería difícil que diferencias en sus niveles de actividad física explicaran los cambios encontrados en marcadores metabólicos. Similarmente, no se midieron patrones alimentarios, pero se instruyó a mantener estos patrones durante la intervención.

En conclusión y considerando la elevada prevalencia de diabetes, sobrepeso y obesidad ${ }^{1}$, en conjunto con los altos niveles de sedentarismo ${ }^{7}$ de la población en Chile, y los elevados costos en tratamiento médico, clínico y farmacológico que conllevan estas alteraciones metabólicas (estimados en más de 471 billones de dólares a nivel mundial ${ }^{22}$, es importante diseñar y considerar estrategias alternativas para mejorar las condiciones de salud en la población. Esta investigación evidencia la efectividad de un programa de EF no tradicional, de bajo costo y alta aplicabilidad, que podría transformarse en una intervención costo-eficiente para la reducción de riesgo cardiovascular, más aun considerando que una de las mayores limitantes para la práctica de EF regular en la población es la falta de tiempo.

Agradecimientos: Queremos agradecer a Carmen Gloria Flores, directora CESFAM Los Lagos, por su permanente preocupación en la promoción de la salud. Al profesor Mario Negrón, Director del Departamento de Ciencias de la Actividad Física de la Universidad de Los Lagos, por su apoyo al proyecto.

\section{Referencias}

1. Minsal. Ministerio de Salud. Gobierno de Chile. Encuesta Nacional de Salud ENS Chile 2009-2010. Disponible en: http://www.redsalud.gov.cl/portal/url/item/99c12b8 9738d80d5e04001011e0113f8.pdf 2011.

2. NCEP. EXecutive summary of the third report of the national cholesterol education program (ncep) expert panel on detection, evaluation, and treatment of high blood cholesterol in adults (adult treatment panel iii). JAMA: The Journal of the American Medical Association 2001; 285 (19): 2486-97.

3. Celis-Morales CA, Pérez-Bravo F, Ibañes L, Sanzana R, Hormazábal E, Ulloa N, et al. Insulin resistance in Chileans of European and indigenous descent: evidence for an ethnicity $\mathrm{x}$ environment interaction. PloS one 2011; 6 (9): e24690. 
4. Fradkin J, Rodgers GP. The Economic Imperative to Conquer Diabetes. Diabetes Care 2008; 31 (3): 624-5.

5. Jenkins N, Hagberg JM. Aerobic Training Effects on Glucose Tolerance in Prediabetic and Normoglycemic Humans. Med Sci Sports Exerc 2011; 43 (12): 2231 40.

6. Andersen JL, Schjerling P, Andersen LL, De la F. Resistance training and insulin action in humans: effects of de-training. The Journal of Physiology 2003; 551 (3): 1049-58.

7. IND. Encuesta nacional de hábitos de actividad física y deporte en la población chilena igual o mayor a 18 años In: Deportes INd, editor. Santiago: Universidad Alberto Hurtado; 2007. p. 1-15.

8. Kessler H, Sisson SB, Short KR. The Potential for HighIntensity Interval Training to Reduce Cardiometabolic Disease Risk. Sports Medicine 2012; 42 (6): 489-509.

9. Gibala MJ, Little JP, MacDonald MJ, Hawley JA. Physiological adaptations to low-volume, high-intensity interval training in health and disease. The Journal of Physiology 2012; 590 (5): 1077-84.

10. Celis-Morales CA, Pérez-Bravo F, Ibáñez L, Salas C, Bailey ME, Gill JM. Objective vs self-reported physical activity and sedentary time: effects of measurement method on relationships with risk biomarkers. PloS one 2012; 7 (5): e36345.

11. WHO. Definition, Diagnosis and Classification of Diabetes Mellitus and its Complications; Report of a WHO Consultation Part 1: Diagnosis and Classification of Diabetes Mellitus. WHO. 1999.

12. Álvarez C, Ramírez R, Flores M, Zúñiga C, Celis-Morales CA. Efectos del ejercicio físico de alta intensidad y sobrecarga en parámetros de salud metabólica en mujeres sedentarias, pre-diabéticas con sobrepeso u obesidad. Rev Med Chile 2012; 140 (10): 1289-96.

13. Marfell-Jones M, Olds T, Stewart A, and Carter L. International standards for anthropometric assessment: ISAK 1st ed. Potchefstroom, South Africa: The International Society for the Advancement of Kinanthropometry (ISAK); 2006.
14. Ciolac EG, Bocchi EA, Bortolotto LA, Carvalho VO, Greve JMD, Guimaraes GV. Effects of high-intensity aerobic interval training vs moderate exercise on hemodynamic, metabolic and neuro-humoral abnormalities of young normotensive women at high familial risk for hypertension. Hypertens Res 2010; 33 (8): 836-43.

15. Friedewa WT, Fredrick DS, Levy RI. Estimation of concentration of low-density lipoprotein cholesterol in plasma, without use of preparative ultracentrifuge. Clinical Chemistry 1972; 18 (6): 499-\&.

16. Karvonen J, Vuorimaa T. Heart rate and exercise intensity during sports activities. Practical application. Sports Med 1988; 5 (5): 303-11.

17. Balducci S, Zanuso S, Cardelli P, Salvi L, Bazuro A, Pugliese L, et al. Effect of High- versus Low- Intensity Supervised Aerobic and Resistance Training on Modifiable Cardiovascular Risk Factors in Type 2 Diabetes; The Italian Diabetes and Exercise Study (IDES). PloS one 2012; 7 (11).

18. Little JP, Safdar A, Wilkin GP, Tarnopolsky MA, Gibala MJ. A practical model of low-volume high-intensity interval training induces mitochondrial biogenesis in human skeletal muscle: potential mechanisms. The Journal of Physiology 2010; 588 (6): 1011-22.

19. Little JP, Gillen JB, Percival ME, Safdar A, Tarnopolsky MA, Punthakee Z, et al. Low-volume high-intensity interval training reduces hyperglycemia and increases muscle mitochondrial capacity in patients with type 2 diabetes. Journal of applied physiology (Bethesda, Md: 1985). 2011; 111 (6): 1554-60.

20. Blair SN, Kohl HW, Gordon NF, Paffenbarger RS. How Much Physical Activity is Good for Health? Annual Review of Public Health 1992; 13 (1): 99-126.

21. Gill JMR, Malkova D. Physical activity, fitness and cardiovascular disease risk in adults: interactions with insulin resistance and obesity. Clinical science (London, England: 1979). 2006; 110 (4): 409-25.

22. Guariguata L. By the numbers: New estimates from the IDF Diabetes Atlas Update for 2012. Diabetes Res Clin Pract 2012; 98 (3): 524-5. 